Cataract Surgery and Lens Implant Surgery J. J. Kanski and R. B. S. Packard. Churchill Livingstone

This short didactic manual gives an account of the authors' current techniques of cataract extraction, lens implantation and combined cataract and glaucoma surgery. In addition there is a chapter on the recognition and management of post-operative complications. The numerous colour illustrations and line drawings are generally of a high standard and complement the well-presented text. The book is recommended for those surgeons who are beginning to learn intraocular surgery.

M G Kerr Muir

Ocular Immunology Gilbert Smolin and Richard O'Connor. Little Brown and Company, Boston

This new edition has been expanded and the presentation improved. The first part concerns a detailed account of the principles of immunology where the advances are now so rapid that it requires the help of summaries such as this to guide those who are interested. The book is basically concerned with clinical immunology of the eye and details the current views of treatment of those ocular diseases which are of great interest and concern to ophthalmologists because of the considerable threat to vision that they may represent. The book concerns the spectrum of disease which is seen in Western societies and does not venture into the problems which are posed in the Third World where much of the infective disease of the eye may result from various kinds of immunodeficiency.

However, there are excellent sections devoted to, for example, amyloidosis, phlyctenulosis, corneal graft rejection and the systemic disorders which are associated with eye disease.

This new edition is a welcome one and provides a considerable insight into visual mechanisms of a series of major diseases of the eye, which would not have been possible even a decade ago. The presentation is clear, and readable and the references, which are mostly from the American literature, are numerous. The authors, who hail from the Proctor Institute in San Francisco, are to be congratulated on this excellent volume which is highly recommended to ophthalmologists who are interested in the mechanisms of an important group of ocular diseases and in particular their treatment which often may present quite major management decisions.

D L Easty

\section{Diagnosis and Management of Ocular Motility}

Disorders. Joyce Mein \& Brian Harcourt. Blackwell Scientific Publications

This book, which is written by two well known clinical practitioners, was never intended to be a comprehensive textbook, but more a practical manual for those who are, or intend to be involved in squint management which may be thought of as one of the more arcane areas of ophthalmology.

The style is didactic, reading rather like an examination primer with each of the three main sections divided into sub-sections and the sub-sections split into further sections which are usually numbered. Whilst this might seem to be a rather inelegant way to present material it is highly effective, especially as the authors have been careful to explain things well and concisely, and not to leave the reader in mid-air having given just a title to the section with no explanation.

It is good to see a sensible proportion of the book devoted to refraction, even if some of it is wasted on the quaint sub-division of hypermetropia into fives types. The rest of the section is excellent with useful guidelines on the prescription and fitting of spectacles-so often this most essential part of ophthalmology is overlooked because it is thought mundane or demeaning for the Ophthalmologist; not here!

The sections on ocular movements, ocular deviation and binocular function are excellent; they are concise, brief, practical and are evidence of the authors practical and commonsense approach. I was amused to note that they devote nearly a quarter of the deviation section to what is obviously the orthoptists favourite pastime - the measurement of 
the $\mathrm{AC} / \mathrm{A}$ ratio-all this in a book so up to date that it does not mention the word squint!

The middle sections on non-surgical and surgical management follow in the same concise manner and I was very impressed with the clear description of complications and the suggestions that were made about the management, although rather strangely they did not even mention in hushed tones the two worst problems-anaesthetic complications including death and the problem of the wrong operation-both are complications which experienced squint surgeons will encounter, and the latter being a problem which one internationally famous squint surgeon has encountered six times! The real meat of the book is in the final section entitled 'Clinical Features, Aetiology and Management of Specific Disorders.' This takes up half of the book and contains the essence of the authors' combined experience in squint management. Despite the terse style it makes good reading and is very practical. Some of the areas where they border on systemic disease the text is a little confusing; for instance it seems to be suggested that the principal weapon in the diagnosis of progressive external ophthalmoplegia is the Hess screen! There is also a section on nystagmus which emphasises surgical treatment three and a half times as much as it does on non-surgical treatment and fourteen times as much time as it spends on differential diagnosis of congenital nystagmus and sensory nystagmus acquired in infancyodd for a method of treatment that may be thought to have questionable longterm value in many cases.

Now as a reviewer, I must 'come clean' with my own readers; if I have sounded the slightest bit unenthusiastic about the book I am afraid its because I am plainly jealous! I am jealous because it is a book on a subject in which I am deeply interested and yet I know I could not have written it as well; jealous because it will sell well to those who do not know much about squint (sorry to use that word in my review) and to those who do. It is an excellent book and it deserves to do well as it is destined to!

Just one question, why is the title 'Ocular Motility Disorders' when the conditions most commonly relate to the eye position not movement; is this the quickening pace of 'advance' away from the time honoured squint? I wonder what the pragmatic Claud Worth would have thought - but he did not have to keep an eye on the American market!

D S I Taylor 